\title{
Efeito da prática no planejamento de ações motoras de indivíduos idosos
}

\author{
Flávio H. Bastos \\ Andrea M. Freudenheim \\ Suely dos Santos
}

Laboratório de Comportamento M otor (Lacom)

Escola de Educação Física e Esporte

Universidade de São Paulo

https://doi.org/10.5628/rpcd.07.01.116

\section{RESUMO}

O objetivo do estudo foi investigar o efeito da prática no planejamento de ações motoras de indivíduos idosos. Participaram do estudo 21 idosos entre 70 e 78 anos de idade. A tarefa consistiu na inserção de uma barra com extremidade semicircular no orifício de uma caixa. Os idosos realizaram, respectivamente, 40, 20 e 20 tentativas nas fases de aquisição, transferência e retenção da transferência. Na aquisição, a barra foi posicionada com sua extremidade voltada para o lado esquerdo e, na transferência e retenção da transferência, para o lado direito. $\mathrm{O}$ momento em que a barra foi orientada corretamente (preensão, transporte e/ou inserção) e o tempo de realização da tarefa foram utilizados como medidas, respectivamente, da qualidade do planejamento e do desempenho. Nas mudanças de fase (aquisição/transferência/retenção da transferência), foi constatada manutenção do planejamento e piora do desempenho. Em função da prática, houve melhora do planejamento ao longo da fase de transferência e melhora do desempenho, ao longo das três fases do experimento $(\mathrm{p}<0,05)$. Concluiu-se que: a capacidade de planejar dos idosos não é tarefa-específica; diante de uma perturbação, idosos trocam velocidade por planejamento; e, que a prática precedida por uma perturbação (modificação da tarefa), desencadeia melhora qualitativa do planejamento.

Palavras-chave: idoso, planejamento de ações motoras, aprendizagem motora

\section{ABSTRACT \\ Effect of practice on elderly's people motor action planning}

The purpose of this study was to investigate the effect of practice on elderly's people motor action planning. Twenty-one elderly aged between 70 and 78 years participated in the study. The task was to insert a rod with a semicircular end in a case hole. The elderly made, respectively, 40, 20 and 20 trials on acquisition, transfer and retention phases. In the acquisition phase, the rod was positioned with its end to the left side and in transfer and retention phases, to the right side. The moment at which the bar was correctly oriented (prehension, transport and/or insertion) and the time spent on the task were considered, respectively, as planning quality and performance measures. As learning progress (acquisition/ transfer/retention), planning quality level was maintained and performance worsened. Planning improved along transfer phase and performance improved along the three experimental phases $(p<0,05)$ due to practice. It may be concluded that: elderly people's ability to plan is not task specific; when facing perturbation, this age group tends to exchange speed for planning and that perturbation (task modification) prior to practice leads to planning quality improvement.

Key-words: elderly, motor action planning, motor learning 


\section{INTRODUÇÃO}

Ao longo do processo de envelhecimento ocorre a deterioração dos sistemas relacionados à execução de habilidades motoras (13, 12). Em consequência, a despeito da pregressa aprendizagem de diversas habilidades, o desempenho motor de indivíduos idosos tende a deteriorar (11, 4). Entretanto, a capacidade de movimentar-se é essencial ao ser humano, pois é a forma através da qual interage no meio ambiente. Mais especificamente, no cotidiano, uma gama de habilidades motoras é requerida, por exemplo, para deslocar-se de um local a outro, para alcançar e apreender objectos em tarefas como escrever e alimentar-se. Portanto, o domínio de habilidades motoras é uma condição necessária à locomoção, comunicação e alimentação independente. Assim, mesmo diante da deterioração resultante do envelhecimento, é impreterível que os idosos mantenham relativa capacidade de solucionar problemas motores, pois sua autonomia para interagir depende em grande parte da mesma (9).

Uma forma de sobrepujar as perdas decorrentes do processo de envelhecimento é lançar mão de estratégias que as compensem (14). Por isso os idosos simplificam as tarefas através da divisão das mesmas em partes, trocam velocidade por precisão e, principalmente, planejam suas acções com antecedência (14). Uma evidência nesse sentido é um estudo no qual se comparou o desempenho de dactilógrafos idosos e jovens ${ }^{(8)}$. Verificou-se que mesmo apresentando tempo de reacção superior ao dos jovens, os idosos foram capazes de dactilografar tão rápido quanto eles. Segundo o autor, isso foi possível devido à estratégia utilizada pelos idosos de antecipar a leitura de palavras enquanto continuavam a dactilografar. Antecipando, eles puderam planejar suas acções motoras e compensar as perdas decorrentes do processo de envelhecimento, mais especificamente, as associadas à solução de problemas motores.

Resultados semelhantes foram observados num estudo, no qual, idosos com tempo de reacção alto foram submetidos à aprendizagem de uma tarefa de timing antecipatório e, através da prática, foram capazes de desenvolver estratégias que compensaram a lentidão para o início da resposta motora (10). De forma geral, planejamento está relacionado a uma acção futura e focaliza o como alcançar uma determinada meta (3). Em relação a acções motoras, a função principal do planejamento é seleccionar, das representações de acção disponíveis, aquelas que serão executadas, relacionando-as a dicas internas e externas e organizando-as na sequência apropriada em função de uma meta específica (2). Entretanto, o planejamento não pode ser directamente observado, mas deve ser inferido através da verificação do comportamento seleccionado (7).

As evidências mais conhecidas sobre planejamento de acções motoras dizem respeito à relação proporcional entre o tempo de reacção e a complexidade das tarefas (2). Mas, esta relação, que resulta no índice de planejamento (index of planning), não fornece evidência directa sobre o que foi planejado e sobre se o tempo de reacção foi utilizado efectivamente para o planejamento. Além disso, não expressa uma característica do próprio movimento, mas do período que o precede. Assim, há poucos estudos na literatura sobre o que planejamos, quanto antecipadamente planejamos e quão bons são esses planos (1). Mais recentemente, considerando a constatação de Haggard (1), Moreira (6) fez inferências sobre a qualidade do planejamento de acções motoras a partir da verificação do comportamento seleccionado. O objectivo do estudo foi investigar o efeito da prática no planejamento de acções motoras de crianças. A tarefa consistiu em inserir uma barra com extremidade semicircular em uma caixa com orifício também semicircular. Para ter sucesso o indivíduo deve orientar a barra segundo o orifício de inserção. Essa tarefa é composta por três fases que compreendem as acções de preensão, transporte e inserção, executadas, necessariamente, nessa sequência (5). Vale ressaltar que a orientação da barra pode ocorrer em qualquer uma das fases da tarefa. Assim, a fase em que a barra é orientada pode servir como medida para auferir o quão antecipadamente as crianças planejaram a inserção. Nesse sentido, a tarefa utilizada permitiu fazer inferências sobre a qualidade do planejamento.

Participaram do experimento 30 crianças, distribuídas em três grupos conforme faixa etária: quatro, cinco e seis anos de idade. O experimento compreendeu as fases de aquisição, transferência e retenção da transferência. Por meio de filmagem, foram obtidas a frequência de tentativas com correcção do 
movimento (medida quantitativa do desempenho) e as fases em que ocorreu a orientação da barra (medida qualitativa do planejamento). Segundo os resultados, foi detectada melhora de desempenho das crianças dos três grupos, mas não foi verificado efeito da prática no planejamento da acção motora em nenhuma das fases do experimento. Uma possível explicação é o fato de crianças, na faixa etária estudada, possuírem poucos recursos cognitivos relacionados à antecipação e planejamento de suas acções.

Idosos possuem os recursos cognitivos necessários ao planejamento e inclusive lançam mão dos mesmos para compensar perdas decorrentes do processo de envelhecimento ${ }^{(8)}$. Nesse sentido, o planejamento de acções motoras assume importante papel no cotidiano dessa população. No entanto, faltam estudos que investiguem a possibilidade de melhora da qualidade do planejamento de acções motoras, e com isso, da qualidade de vida da população idosa. Assim, o objectivo do presente estudo foi investigar o efeito da prática no planejamento de acções motoras de idosos a partir de um enfoque qualitativo sugerido por Haggard (1998).

\section{MÉTODO}

\section{Amostra}

Participaram do estudo 21 indivíduos idosos entre 70 e 78 anos de idade ( $x=72,4$ anos; $s=2,33$ ), frequentadores do programa "Atividade Física para a Terceira Idade" do Centro de Práticas Esportivas da Universidade de São Paulo. Todos os voluntários assinaram um termo de consentimento livre e esclarecido aprovado pela Comissão de Ética e Pesquisa da Escola de Educação Física e Esporte da Universidade de São Paulo.

\section{Equipamento}

O equipamento utilizado consistiu de: a) uma caixa de madeira $\left(A=25 \mathrm{~cm}^{2}, \mathrm{~h}=4,5 \mathrm{~cm}\right)$ com orifício semicircular no centro da tampa (Figura 1); b) uma barra de madeira $(\mathrm{d}=4 \mathrm{~cm}$, peso $=300 \mathrm{~g})$, com uma porção cilíndrica $(\mathrm{c}=10 \mathrm{~cm})$ e uma porção semicilíndrica, denominada extremidade $(\mathrm{c}=5 \mathrm{~cm})$, de cor vermelha; c) um cavalete de madeira $(\mathrm{h}=6,5 \mathrm{~cm}$ no ponto de tangência com a barra); uma câmera de vídeo S-VHS; e, g) duas lâmpadas, uma verde e a outra laranja. Sobre a mesa ficaram alinhados, à frente do partici- pante, o cavalete com a barra apoiada horizontalmente sobre ele e, logo adiante, a caixa. A extremidade da barra foi colocada com a face rectilínea paralela à mesa e a parte curvilínea voltada para cima.

\section{Tarefa e procedimentos}

Em função do objectivo do estudo optou-se por utilizar a tarefa de Moreira (6), que consistiu na inserção da barra com extremidade semicircular no orifício, também semicircular de uma caixa de madeira. A tarefa é composta por três fases. A fase de preensão que compreende, em sequência, a retirada da mão da mesa, alcançar e segurar a barra; a fase de transporte, que tem início com a movimentação da barra para retirá-la do cavalete e finaliza com sua condução às proximidades da caixa; e, a fase de inserção, que inicia com o contato da barra com a tampa da caixa e finaliza com a inserção completa da barra no orifício.

A execução da tarefa ocorreu mediante a seguinte instrução do experimentador: "Fique com as mãos sobre a mesa. Quando a luz verde acender, você deverá segurar a barra pela parte verde e inserir a extremidade vermelha no orifício da caixa à sua frente. A luz laranja acenderá quando você finalizar a ação. Serão repetidas várias tentativas".

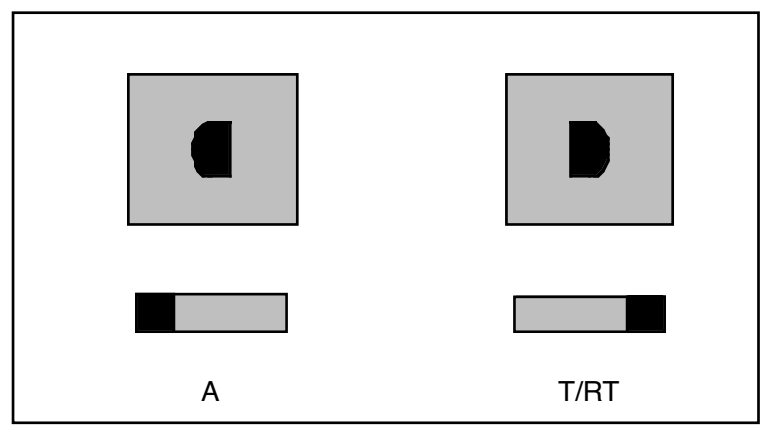

Figura 1. Representação esquemática do instrumento com o posicionamento dos orifícios e da barra nas fases de Aquisição (A) e Transferência e Retenção da Transferência [T/RT].

\section{Delineamento}

O experimento consistiu de três fases: aquisição (A), transferência $(\mathrm{T})$ e retenção da transferência $(\mathrm{RT})$, sendo que, nesta última, procurou-se verificar se a capacidade de generalização da habilidade adquirida foi duradoura. 
Foram realizadas quarenta tentativas na fase de aquisição, vinte no teste de transferência e mais vinte no teste de retenção da transferência. Na fase de aquisição, a barra foi posicionada com sua extremidade voltada para o lado esquerdo e, nos testes de transferência e retenção da transferência, para o lado direito. Da fase de aquisição para as de transferência e retenção da transferência, mudou-se também o posicionamento do orifício (vide Figura 1). O teste de transferência foi realizado após a fase de aquisição e o teste de retenção da transferência, dois dias após as fases de aquisição e transferência.

\section{Medidas e análise dos dados}

Em função da natureza do estudo optou-se por utilizar uma medida qualitativa, relativa ao planejamento da acção e uma medida quantitativa, de desempenho na tarefa. A medida qualitativa derivou da(s) fase(s) ou momentos em que o participante efectuou a orientação da barra (6). Nesse sentido, os comportamentos foram classificados em: $\mathrm{C} 1$ - fase de preensão; C2 - ao longo das fases de preensão e transporte; C3 - fase de transporte; C4 - ao longo das fases de transporte e inserção; C5 - ao longo das três fases; e, C6 na fase de inserção (Tabela 1).

Tabela 1. Fase [s] em que ocorreu orientação da barra e respectiva denominação do comportamento.

\begin{tabular}{lc}
\hline Fase(s) & Comportamento \\
\hline Preensão (com manipulação) & $\mathrm{C} 1$ \\
Preensão e transporte & $\mathrm{C} 2$ \\
Transporte & $\mathrm{C} 3$ \\
Transporte e inserção & $\mathrm{C} 4$ \\
Preensão, transporte e inserção & $\mathrm{C} 5$ \\
Inserção & $\mathrm{C} 6$ \\
\hline
\end{tabular}

Foi estabelecido que a fase em que o participante inicia a orientação da barra indica quão antecipadamente este começou a planejar a solução do problema (inserção da barra); por sua vez, o momento em que o participante soluciona a orientação, foi utilizado como indicativo de quão antecipadamente a inserção foi correctamente planejada. Nesse sentido, em termos de planejamento da acção motora, os comportamentos de C1 a C6 estão sequenciados no sentido de qualitativamente superior (melhor) para qualitativamente inferior (pior).
A medida de desempenho utilizada foi o tempo de movimento, ou seja, o tempo compreendido entre a retirada da mão da mesa e a inserção completa na extremidade da barra. Esse tempo foi calculado através da razão entre o número de quadros na imagem e a frequência do aparelho de SVHS utilizado $(30 \mathrm{~Hz})$. Os resultados obtidos foram agrupados em blocos de cinco tentativas (média para o tempo de movimento e moda para os comportamentos observados). Dessa forma, as fases de aquisição, transferência e retenção da transferência correspondem a oito, quatro e quatro blocos de tentativas, respectivamente.

A análise qualitativa do comportamento foi realizada através do teste não-paramétrico de Friedman (medidas repetidas intra-grupo), e do teste de Wilcoxon corrigido pelo procedimento de Bonferroni (para localização das diferenças). Já a análise inferencial dos dados referentes ao tempo de movimento foi realizada mediante aplicação da ANOVA one-way para medidas repetidas e do teste de Tukey (post hoc).

\section{RESULTADOS}

A análise dos resultados visou verificar se: (a) houve mudança nos comportamentos/tempos de movimento utilizados ao longo da fase de aquisição; (b) houve manutenção dos comportamentos/tempos de movimento exibidos do final da fase de aquisição para a fase de transferência; e, (c) os comportamentos/tempos de movimento exibidos na fase de transferência se mantiveram na fase de retenção da transferência. Os resultados relativos aos comportamentos (qualidade do planejamento) e os tempos de movimento (desempenho) dos idosos estão apresentados, respectivamente, nas Figuras 2 e 3.

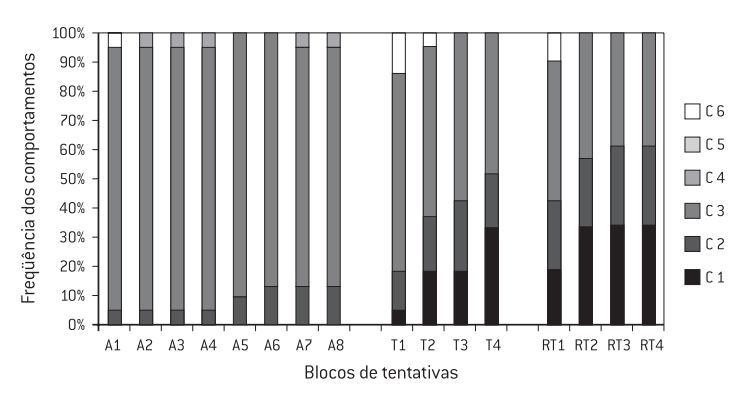

Figura 2. Distribuição da freqüência (moda) dos comportamentos exibidos pelos idosos ao longo das fases de aquisição $(A)$, transferência $(T)$ e retenção da transferência (RT). 
O teste de Friedman não detectou diferenças significantes na frequência dos comportamentos exibidos ao longo da fase de aquisição ( $\mathrm{X}^{2}[\mathrm{~N}=21, \mathrm{gl}=7]$ $=5,182 \mathrm{p}<0,638)$. Já na comparação entre o final da fase de aquisição e as fases de transferência e retenção da transferência foi encontrada diferença significante $\left(\mathrm{X}^{2}[\mathrm{~N}=21, \mathrm{gl}=8]=35,747 \mathrm{p}<\right.$ $0,001)$. Foi realizado um teste de Wilcoxon com correcção pelo procedimento de Bonferroni, a partir do qual o índice de significância passou a ser $\mathrm{p}<0,00143$. Esse procedimento não possibilitou a localização das diferenças encontradas pelo teste de Friedman. O valor de $Z$, encontrado pelo teste de Wilcoxon, sugere que as diferenças estejam localizadas entre o primeiro bloco da fase de transferência (T1) e os blocos T4 (último da fase de transferência), RT2, RT3 e RT4 (segundo, terceiro e quarto blocos da fase de retenção da transferência).

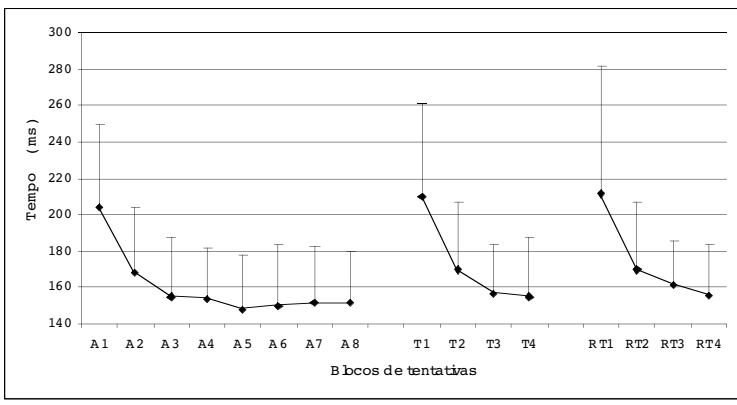

Figura 3. Médias e desvios padrão do tempo de movimento exibidos pelos idosos ao longo das fases de aquisição $(A)$, transferência $(T)$ e retenção da transferência [RT].

A ANOVA one-way utilizada para analisar a fase de aquisição apontou diferença significante ( $\mathrm{F}[7$, $140]=32,014, \mathrm{p}<0,001)$, localizada pelo teste de Tukey entre o primeiro bloco (A1) e todos os demais $(\mathrm{p}<0,001)$ e o segundo bloco (A2) e todos os demais $(\mathrm{p}<0,05)$, com excepção da comparação A2A3. Na comparação entre o último bloco da fase de aquisição e os blocos das fases de transferência e retenção da transferência a ANOVA one-way também encontrou diferenças significantes ( $\mathrm{F}[8$, $160]=11,933, \mathrm{p}<0,001)$. A análise post hoc realizada através do teste de Tukey localizou as diferenças entre o último bloco da fase de aquisição e o primeiro bloco das fases de transferência e retenção da transferência $(p<0,001)$; entre o primeiro bloco da fase de transferência e todos os demais $(\mathrm{p}<0,001)$, com excepção do primeiro bloco da fase de retenção da transferência; e entre o primeiro bloco da fase de retenção da transferência e todos os demais $(p<0,001)$, com excepção do primeiro bloco da fase de transferência.

\section{DISCUSSÃO}

O objectivo do estudo foi investigar o efeito da prática no planejamento de acções motoras de idosos. Nesse sentido, a análise dos resultados visou verificar se: (a) houve mudança nos omportamentos/tempos de movimento utilizados ao longo da fase de aquisição; (b) houve manutenção dos comportamentos/tempos de movimento exibidos do final da fase de aquisição para a fase de transferência; (c) os comportamentos/tempos de movimento exibidos na fase de transferência se mantiveram na fase de retenção da transferência. A discussão a seguir remete a cada uma dessas questões e, por isso, está organizada em função das fases do experimento.

\section{Fase de aquisição}

Ao longo da fase de aquisição, mesmo após 40 tentativas de prática com liberdade para em que fase da tarefa orientar a barra, não ocorreu mudança significante nos comportamentos exibidos pelos idosos. Houve predominância do $\mathrm{C} 3 \mathrm{em}$ todos os blocos, ou seja, na maioria das tentativas os idosos iniciaram e finalizaram a orientação da barra na fase de transporte (Figura 2). Nesse sentido, em conformidade com a literatura $(8,13,14)$, pode-se inferir que os idosos planejam e solucionam suas acções com boa antecedência, no entanto, a prática não resultou na melhora na qualidade do planejamento, ao menos quando é analisada na mesma tarefa.

Por sua vez, os idosos melhoraram seu desempenho em função da prática, pois nos dois primeiros blocos da fase de aquisição demoraram mais para realizar a tarefa que nos demais (excepção entre A2-A3). Essa melhora no desempenho pode ser explicada em função do tempo despendido no planejamento das acções. Pode-se supor que, em função da prática, o tempo para efectuar o planejamento diminuiu. Nesse sentido, ao longo da fase de aquisição, os idosos 
mantiveram a estratégia (C3), mas diminuíram o tempo para planejá-la e, em consequência, houve redução significante do tempo para realizar a tarefa. Assim, pode-se inferir que os idosos são conservadores em relação às estratégias dominadas.

\section{Fase de transferência}

Como do final da fase de aquisição (A8) para o início da fase de transferência (T1) houve pouca modificação na distribuição dos comportamentos, inferese que, nesse quesito, os idosos não foram sensíveis à modificação da tarefa (inversão da barra e da orientação do orifício da caixa). Portanto, idosos conseguem planejar suas acções com boa antecedência e transferem essa estratégia para tarefas correlatas, ou seja, conseguem generalizar o processo de solução de problemas motores. Assim sendo, em se tratando de idosos, a capacidade de planejar não é tarefaespecífica, pois tarefas distintas (fase de aquisição/fase de transferência) são solucionadas através da mesma estratégia de planejamento. Porém, chama a atenção que o C6, que é considerado um comportamento qualitativamente inferior, extinto na fase de aquisição, volta a ser exibido nos primeiros blocos da fase de transferência. Essa tendência de piora do planejamento indica que o poder de generalização da estratégia não é ilimitado. Seria relevante, em estudos futuros, explorar o limite dessa abrangência.

Em termos do desempenho, houve uma deterioração na passagem da fase de aquisição (A8) para a de transferência (T1). Isso significa que a modificação da tarefa gerou uma perturbação, pois em função da mesma, os idosos passaram a demorar mais para realizar a tarefa. Esse decréscimo do desempenho não pode ser explicado a partir de mudanças na distribuição dos comportamentos, pois o mesmo foi mantido de A8 para T1. Uma possível explicação é a de que os idosos optaram por manter a estratégia em detrimento do tempo de execução da tarefa. Em outras palavras, embora em A8 e T1 tenham planejado e solucionado o problema nas mesmas fases da tarefa, em T1 demoraram mais para planejar e, em consequência, despenderam mais tempo para executar a tarefa que em A8.

Portanto, em conjunto, os resultados da comparação entre o último bloco da fase de aquisição (A8) e o primeiro da fase de transferência (T1) mostram que, frente a uma perturbação (tarefa modificada), os idosos são capazes de manter uma boa estratégia de planejamento, portanto, de generalizá-la, mas que para isso precisam aumentar o tempo de realização da mesma. Ainda, que frente a novos desafios, além de trocar velocidade por precisão $(13,14)$, idosos trocam velocidade por planejamento estratégico dominado. Por sua vez, como uma diferença se localiza, provavelmente, entre os blocos inicial e final da fase de transferência (T1 e T4), pode-se inferir que, diferentemente de resultados encontrados na literatura (6), a prática quando precedida de uma perturbação (modificação da tarefa) resulta em melhora da qualidade do planejamento, pois desencadeia a antecipação do planejamento por parte dos idosos. Mais especificamente, ao longo da fase de transferência há presença crescente do $\mathrm{Cl}$ (comportamento considerado qualitativamente superior), substituindo, num primeiro momento o $\mathrm{C} 6$ ( $\mathrm{T} 1$ e $\mathrm{T} 2$ ) e, em um segundo momento, o C3 (T1-T2 / T3-T4). Essa melhora culmina no $\mathrm{T} 4$, quando os idosos passam a orientar a barra na preensão ( $\mathrm{C} 1$ e C2) em $50 \%$ das execuções, ou seja, em $30 \%$ a mais das execuções que no primeiro bloco da fase de transferência (T1).

No que se refere ao desempenho, os resultados mostraram que houve melhora ao longo da fase de transferência. Ante este fato, pode-se supor que a antecipação do planejamento resultou na diminuição do tempo despendido na realização da tarefa. Mas, essas mudanças não ocorreram na mesma razão. Em relação ao comportamento foi detectada diferença significante somente entre T1 e T4. Já em relação ao desempenho, houve melhora significante entre todos os blocos de tentativas. Nesse sentido, pode-se inferir que, as mudanças na qualidade do planejamento são relativamente mais lentas que as mudanças no desempenho. Assim, em estudos futuros deve-se considerar a possibilidade de um delineamento que compreenda uma prática extensiva.

\section{Fase de retenção da transferência}

A ocorrência de poucas mudanças comportamentais do final da fase de transferência (T4) para o início da fase de retenção da transferência (RT1), mostra que o intervalo de dois dias não prejudicou a qualidade do planejamento dos idosos. No entanto, em função 
do intervalo, houve tendência de piora da qualidade do planejamento - T1 é diferente de RT2, RT3 e RT4, mas não de RT1; e, em T1, houve diminuição do $\mathrm{C} 1$ e volta de exibição do C6. Mas, com pouca prática adicional mesmo essa tendência desaparece, pois já no segundo bloco da fase de retenção da transferência (R2), os idosos voltaram a substituir o C6 pelo C1. Portanto, idosos retém a qualidade do planejamento resultante da prática, mesmo sem dar continuidade à mesma. Esse resultado é um indicativo forte de que nas fases que antecederam a retenção da transferência houve a formação de uma representação da acção (programa de acção) persistente. Entretanto, o intervalo de dois dias prejudicou o desempenho - da fase de transferência (T4) para a de retenção da transferência (RT1) os idosos passaram a demorar mais para realizar a mesma tarefa - ou seja, gerou uma perturbação. Mas, diante da perturbação, como na passagem da fase de aquisição para a de transferência, os idosos optaram por manter a estratégia em detrimento do tempo de execução da tarefa. Esse aumento no tempo expressa a dificuldade na retomada de uma tarefa anteriormente praticada. Portanto, em conjunto, os resultados da comparação entre o último bloco da fase de transferência (T4) e o primeiro da fase de retenção da transferência (RT1) mostram que, diante do aumento da dificuldade em realizar uma tarefa, os idosos optam por manter as estratégias de planejamento, ou seja, por utilizar um programa de acção já disponível (2), mas para isso precisam aumentar o tempo de realização da mesma. Assim, frente ao aumento da dificuldade de realizar uma tarefa, os idosos também trocam velocidade por planejamento.

Ao longo da fase de retenção da transferência não foram detectadas diferenças significantes relativas à distribuição dos comportamentos. Mas, chama a atenção que a partir do primeiro bloco da fase de retenção da transferência em $60 \%$ das tentativas os idosos iniciaram a busca da solução já na fase de preensão. Destas tentativas, a solução do problema se deu em $38 \%$ na própria fase de preensão e em $22 \%$ na fase seguinte, ou seja, na de transporte. Esses comportamentos ( $\mathrm{C} 1$ e $\mathrm{C} 2$ ), podem ser considerados como sendo, respectivamente, excelente e óptimo, no que se refere à qualidade do planejamento. Assim, os resultados mostram que idosos são capazes de planejar muito bem suas acções, em outras palavras, dada a oportunidade de praticar, idosos são capazes de elaborar estratégias eficientes para resolver uma tarefa motora, corroborando o que foi apontado na literatura (14).

No que se refere ao desempenho, os resultados mostraram que houve melhora ao longo da fase de retenção da transferência. Ante este fato, pode-se supor que o tempo para efectuar o planejamento diminuiu ao longo da fase de retenção da transferência e que essa diminuição, como na fase de aquisição, resultou na melhora do desempenho.

\section{CONCLUSÕES}

O objectivo do estudo foi investigar o efeito da prática no planejamento de acções motoras de idosos. Nesse sentido pode-se concluir que, em uma tarefa de orientação e inserção de uma barra em um orifício de uma caixa:

1. Idosos planejam suas acções com boa antecedência;

2. A capacidade de planejar de idosos não é tarefaespecífica, pois tarefas distintas (fase de aquisição/fase de transferência) são solucionadas através da mesma estratégia de planejamento;

3. Idosos conseguem reter a qualidade do planejamento resultante da prática, mesmo sem dar continuidade à mesma;

4. Diante de uma perturbação, independente se gerada por uma modificação da tarefa (fase de transferência) ou por um intervalo de prática (fase de retenção da transferência), idosos trocam velocidade por planejamento, isto é, preferem manter sua estratégia de planejamento em detrimento da velocidade de realização da tarefa.

5. A prática não resulta na melhora na qualidade do planejamento quando se dá em uma mesma tarefa (fases de aquisição e retenção da transferência). Entretanto, ela desencadeia a melhora qualitativa do planejamento quando precedida por uma perturbação (fase de transferência). Portanto, o efeito da prática no planejamento de acções motoras de idosos depende do contexto da prática.

6. Em função de 4) e 5), pode-se concluir que idosos são conservadores em relação às estratégias de planejamento aprendidas. 
No seu conjunto, os resultados desse estudo complementam os achados da literatura. No entanto, é preciso olhá-los com cautela, pois se trata de um estudo pontual, que utiliza, por exemplo, uma tarefa específica. Nesse sentido, faz-se necessária a realização de estudos adicionais, que abordem o problema, por exemplo, em diferentes tarefas, modificações da tarefa e quantidades de prática.

\section{NOTA}

Pesquisa fomentada pela FAPESP (01/13857-0).

\section{REFERÊNCIAS BIBLIOGRÁFICAS}

1. Haggard P (1998). Planning of action sequences. Acta Psychol (Amst) v.99, p.201-15

2. Jeannerod M (1997). The Cognitive Neuroscience of Action. Cambridge, Massachusetts: Blackwell Publishers

3. Kreitler S, Kreitler H (1987). Conceptions and processes of planning: the developmental perpective. In: Friedman SL, Scholnick EK, Cocking RR (eds.). Blueprints for Thinking: the role of planning in cognitive development. New York: Cambridge University Press, p.205-272.

4. Kretchmar RS (2000). Physical activity, aging, and disability. Quest 52: 331-332.

5. Manoel EJ, Connolly KJ (1997). Variability and stability in the development of skilled actions. In: Connolly KJ, Forssberg H, (eds.) N europhysiology \& neuropsychology of motor development. London: Mac Keith, p.286-318

6. Moreira CRP (2002). Efeito da Prática no Planejamento de uma habilidade de M anipulação em Crianças de Primeira Infância. Dissertação (Mestrado). Escola de Educação Física e Esporte - Universidade de São Paulo, 64 f.

7. Rosenbaum DA, Vaughan J, Barnes HJ, Jorgensen MJ (1992). Time course of movement planning: Selection of hand grips for object manipulation. J Exp Psychol Learn M em Cog 18: 1058-1073

8. Salthouse TA (1984). Effects of the age and skill on typing. J Exp Psychol Gen 113: 345-371

9. Santos S, Dantas L, Oliveira JA (2004). Desenvolvimento motor de crianças, de idosos e de pessoas com transtornos da coordenação. Revista Paulista de Educação Física 18: 33-44

10. Santos S, Tani G (1995). Tempo de reação e aquisição de uma tarefa de timing antecipatório em idosos. Revista Paulista de Educação Física 9(1): 51-62.

11. Santos, S (2002). Desenvolvimento motor ao longo da vida (339-349). In: Barbanti VJ, Amadio AC, Bento JO, Marques AT (Eds.), Esporte e atividade física: interação entre rendimento e qualidade de vida. São Paulo: Manole.

12. Shephard RJ (2003). Envelhecimento, atividade física e saúde. São Paulo: Phorte.

13. Spirduso WW, Francis KL, MacRae P.G. (2005). Physical Dimensions of A ging ( $2^{\mathrm{a}} \mathrm{Ed}$.). Champaign, Illinois: Human Kinetics

14. Welford AT (1985). Changes of performance with age: na overview. In: Charness (Ed.), A ging and human performance (333-369). Chichester: John Wiley \& Sons.

\section{CORRESPONDÊNCIA}

\section{Flávio H. Bastos}

Laboratório de Comportamento Motor (Lacom)

EEFE - USP

Av. Prof. Mello Moraes, 65

CEP 05508-900-São Paulo

SP- BRASIL

e-mail: bastosfh@ usp.br 